\title{
Muon g-2 and dark matter in models with vector-like fermions*
}

\author{
Enrico Maria Sessolo ${ }^{\dagger}$ \\ Technische Universität Dortmund \\ National Centre for Nuclear Research, Warsaw \\ E-mail: enrico.sessolo@ncbj.gov.pl \\ Kamila Kowalska \\ Technische Universität Dortmund \\ National Centre for Nuclear Research, Warsaw \\ E-mail: kamila.kowalska@ncbj.gov.pl
}

\begin{abstract}
We investigate simplified models of new physics that can accommodate the measured value of the anomalous magnetic moment of the muon and the relic density of dark matter. We define a set of renormalizable, $\mathrm{SU}(2) \times \mathrm{U}(1)$ invariant extensions of the Standard Model, each comprising an inert $\mathbb{Z}_{2}$-odd scalar field and one or more vector-like pairs of colorless fermions that communicate to the muons through Yukawa-type interactions. The new sectors are classified according to their transformation properties under the Standard Model gauge group and all models are systematically confronted with a variety of experimental constraints: LEP mass bounds, direct LHC searches, electroweak precision observables, and direct searches for dark matter. We show that scenarios featuring only one type of new fermions become very predictive once the relic density and collider constraints are taken into account, as in this case $(g-2)_{\mu}$ is not enhanced by chirality flip. Conversely, for models where an additional source of chiral-symmetry violation is generated via fermion mixing, the constraints are much looser and new precision experiments with highly suppressed systematic uncertainties may be required to test the parameter space.
\end{abstract}

The European Physical Society Conference on High Energy Physics

5-12 July

Venice, Italy

${ }^{*}$ DO-TH $17 / 24$
${ }^{\dagger}$ Speaker. 


\section{Introduction}

The BNL measurement [1] of the anomalous magnetic moment of the muon, $(g-2)_{\mu}$, shows a discrepancy with the Standard Model $(\mathrm{SM})$ at the $\sim 3.5 \sigma$ level: $\delta(g-2)_{\mu}=(27.4 \pm 7.6) \times$ $10^{-10}$ [2]. The interest in this anomaly is bound to receive a boost by the start of the new Muon g-2 experiment at Fermilab [3], which will improve the statistical precision of the measurement by a factor of four or so with respect to BNL. Additionally, just a few years after the Fermilab experiment, $(g-2)_{\mu}$ will also be measured at J-PARC [4], which is expected to reach a comparable sensitivity even if the experimental setup is different.

In these proceedings we report on our recent paper, Ref. [5], in which we analyzed the implication of the $(g-2)_{\mu}$ measurement for several simplified models of new physics and we derived predictions for future tests. For each model considered, we highlighted the regions of the parameter space able to simultaneously accommodate $\delta(g-2)_{\mu}$ and the relic density of dark matter.

The models we construct are based on the following requirements: 1) the dark matter interacts with the muons through renormalizable couplings; 2) interactions are $\mathrm{CP}$ conserving and invariant under the SM gauge group, $\mathrm{SU}(2) \times \mathrm{U}(1) ; 3)$ each model satisfies the constraints from perturbativity and unitarity; 4) the measurement of the relic abundance is an active constraint on the parameter space. We do not consider in this paper dark matter lighter than the mass of the muon so that, to make it stable, we introduce an additional discrete symmetry, $\mathbb{Z}_{2}$, under which the dark matter is odd and the SM is even.

The first of the requirements listed above limits the allowed interactions to fermion-scalarfermion and fermion-vector-fermion types. As one of the participating fermions is necessarily the muon, the discrete symmetry forces us to additionally introduce $\mathbb{Z}_{2}$-odd colorless fermions, which must be vector-like (VL) to evade the bounds from electroweak precision observables (EWPOs) and to not introduce gauge anomalies. Note that all our assumptions are trivially satisfied by Yukawatype interactions fermion-(pseudo)scalar-fermion, once the appropriate scalar potential is spelled out. For this reason, in our study we limit ourselves to discussing this kind of interactions, leaving the case with vector particles for future work.

\subsection{Overview of analyzed models}

Interaction terms in the Lagrangian of the models we consider are given by

$$
\mathscr{L} \supset\left(g_{s} \bar{\psi}_{E} \psi_{\mu} \phi_{S}+i g_{p} \bar{\psi}_{E} \gamma^{5} \psi_{\mu} \phi_{S}+\text { h.c. }\right)-m_{E} \bar{\psi}_{E} \psi_{E}-V\left(\phi_{S}\right),
$$

in terms of a Yukawa coupling $g_{s}\left(g_{p}\right)$ of the muon to a (pseudo)scalar field $S$ - whose dynamics is described by an appropriate scalar potential $V\left(\phi_{S}\right)$ - and a generic heavy fermion $E$ of mass $m_{E}$.

The specific value of $(g-2)_{\mu}$ depends on the electric charge and spin quantum numbers of the particles running in the loop. Considering, for example, a charged fermion and a neutral scalar, one gets (see, e.g., [6] for a review of the calculation)

$$
\begin{aligned}
\delta(g-2)_{\mu}=\frac{m_{\mu}^{2}}{8 \pi^{2} m_{S}^{2}}\left[\left(\left|g_{s}\right|^{2}+\left|g_{p}\right|^{2}\right)\right. & \int_{0}^{1} d x \frac{x^{2}(1-x)}{(1-x)\left(1-\lambda^{2} x\right)+\varepsilon^{2} \lambda^{2} x} \\
& \left.+\varepsilon\left(\left|g_{s}\right|^{2}-\left|g_{p}\right|^{2}\right) \int_{0}^{1} d x \frac{x^{2}}{(1-x)\left(1-\lambda^{2} x\right)+\varepsilon^{2} \lambda^{2} x}\right],
\end{aligned}
$$




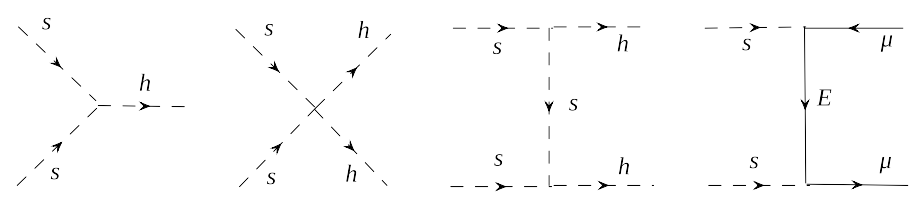

Figure 1: Starting from the left, the first 3 diagrams show the well-known scalar portal interactions that can potentially lead to the correct dark matter relic abundance. The last diagram on the right depicts the $t$-channel "bulk" mechanism via a new heavy fermion $E$.

in terms of the mass ratios of the new particles to the muon, $\varepsilon=m_{E} / m_{\mu}, \lambda=m_{\mu} / m_{S}$.

Given a specific scalar sector, we consider all possible Yukawa interactions allowed by the $\mathrm{SU}(2) \times \mathrm{U}(1)$ and $\mathbb{Z}_{2}$ symmetries that can lead to the $(g-2)_{\mu}$ anomaly by combining the scalar field with VL fermion multiplets. The combinations we investigate are the following, and the details can be found in [5].

1. Models with a real neutral scalar SU(2) singlet: a. Fermion SU(2) singlets; b. Fermion SU(2) doublets; c. Singlet and doublet fermions mixing with each other via the Higgs field vacuum expectation value (vev).

2. Models with a complex scalar SU(2) singlet: a. Fermion SU(2) singlets; b. Fermion SU(2) doublets; c. Singlet and doublet fermions mixing via the Higgs vev.

3. Models with an SU(2) scalar doublet: a. Fermion SU(2) singlets; b. Fermion SU(2) doublets; c. Fermion SU(2) triplets; d. Fermion adjoint triplets; e. Fermions mixing via Higgs vev.

We review in the next sections Cases 1 and 3 above, characterized by models with a real scalar singlet and a scalar doublet. Analysis of the remaining models can be found in [5].

\section{Models with a real neutral scalar singlet}

Let us extend the SM by a neutral real scalar, $s$, singlet under SU(2). For simplicity, we assume in this work that any new introduced scalar is inert, in the sense that it does not develop a vev.

The scalar potential, given in [5], features well-known dark matter properties: the WIMP $s$ efficiently annihilates in the early Universe through the interaction vertices depicted in the first three diagrams of Fig. 1. However, it is also well known (see, e.g., [7]) that the portal coupling and dark matter mass are subject to strong bounds from direct detection searches, which, as we shall see below, after the most recent bound from XENON-1T [8] is taken into account exclude the mass range $m_{\mathrm{DM}}=m_{s} \approx 10-800 \mathrm{GeV}$ if one imposes the relic density constraint. This is precisely the mass range where the new scalar field and VL leptons can positively contribute to $\delta(g-2)_{\mu}$.

Interestingly, the presence of VL fermions opens up additional mechanisms for dark matter annihilation, as shown in the last diagram on the right of Fig. 1. The limits from direct detection searches can be then easily evaded, thanks to the leptophilic nature of the interaction between the dark matter scalar and the SM. By borrowing a term often used in supersymmetry, we will hence refer to this mechanism as the bulk.

In the first class of models (Case 1a in the list of Sec. 1.1) we add to the $\mathrm{SM}+s$ a pair of charged Weyl spinor $\mathrm{SU}(2)$ singlets, odd under $\mathbb{Z}_{2}$. In terms of the heavy Dirac lepton, $\widetilde{E}$, Eq. (1.1) reads $\mathscr{L} \supset-Y_{S} \bar{\psi}_{\widetilde{E}} P_{R} \psi_{\mu} s+$ h.c., so that one has $g_{s}=Y_{S} / 2, i g_{p}=Y_{S} / 2$ and only the first line in Eq. (1.2) gives a contribution to $(g-2)_{\mu}$. 


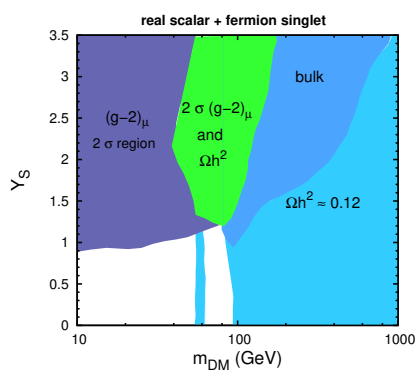

(a)

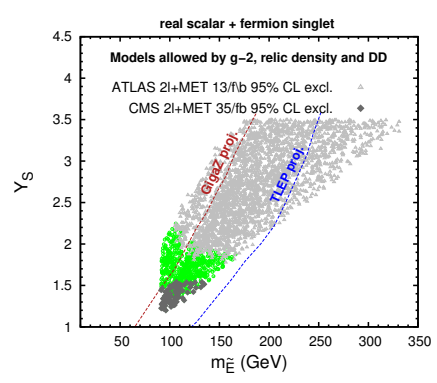

(c)

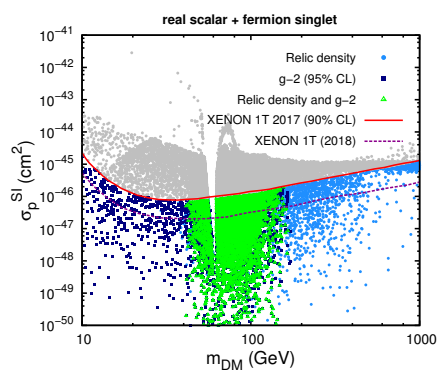

(b)

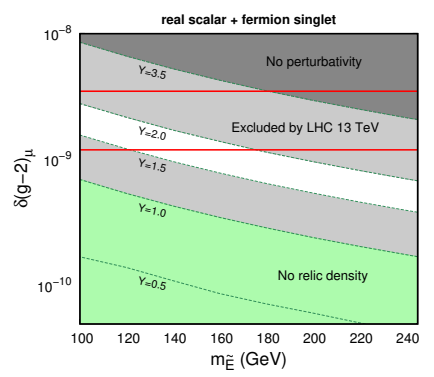

(d)

Figure 2: (a) The $\left(m_{\mathrm{DM}}, Y_{S}\right)$ plane for Case 1a. In cyan, the parameter space favored at $2 \sigma$ by the relic density is shown, while the one favored by the $(g-2)_{\mu}$ measurement is shown in dark blue. Green region corresponds to those values of model parameters where both constraints are satisfied simultaneously. (b) Same as (a), but in the $\left(m_{\mathrm{DM}}, \sigma_{p}^{\mathrm{SI}}\right)$ plane. (c) The parameter space common to the relic density and $(g-2)_{\mu}$ in the $\left(m_{\widetilde{E}}, Y_{S}\right)$ plane. Gray triangles show the parameter space excluded by the ATLAS 2 hard leptons search [9], whereas dim gray diamonds show that excluded by the CMS 2 soft leptons search [10]. The projected reach of precision measurements at GigaZ [11] and TLEP [12] is also shown. (d) The measurement of $\delta(g-2)_{\mu}$ as a function of the VL fermion mass and the new Yukawa coupling. Here $m_{s}=m_{\mathrm{DM}}=80 \mathrm{GeV}$. The horizontal red solid lines show the $2 \sigma$ region of the BNL experiment.

In Fig. 2(a) we present a plot of the model's parameter space in the plane of the new coupling to the muon, $Y_{S}$, versus the dark matter mass, $m_{\mathrm{DM}}=m_{s}$. We do not impose at this stage any LHC or EWPO constraints. The parameter space allowed at $2 \sigma$ by the combination of relic density and $(g-2)_{\mu}$ is shown in green. In Fig. 2(b) we show the allowed parameter space in the $\left(m_{\mathrm{DM}}, \sigma_{p}^{\mathrm{SI}}\right)$ plane. Dark matter due to Higgs-portal couplings plays a small role, almost exclusively limited to the region above $0.8-1 \mathrm{TeV}$, in which the recent bounds from XENON1T can be evaded. Lighter dark matter is due to the bulk mechanism. The points of the allowed parameter space - the green region of Fig. 2(a) - are shown in Fig. 2(c), in the plane of the new Yukawa coupling, $Y_{S}$, versus the VL fermion mass. We have applied here the LHC bounds [9] and [10]. Because of the overall large Yukawa values, future precision experiments like GigaZ or TLEP, with a projected improvement by a factor 20 or more over LEP, have the potential to probe the surviving region. We summarize our findings in Fig. 2(d), where we present predictions for this model based on an eventual measurement of $(g-2)_{\mu}$ at Fermilab.

One can consider alternatives characterized by the coupling of the muon to a fermion doublet (Case $1 \mathrm{~b}$ in the list of Sec. 1.1) and models where the singlet and doublet fermion are both included 


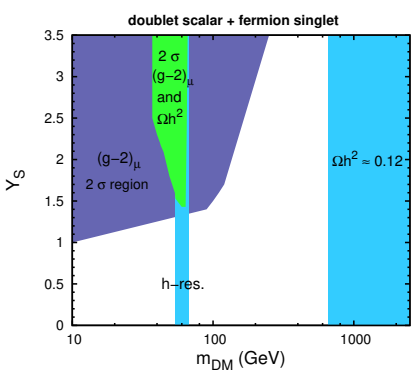

(a)

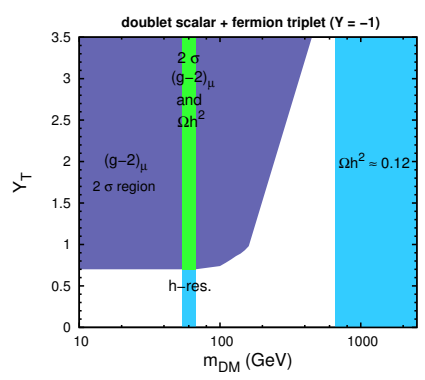

(b)

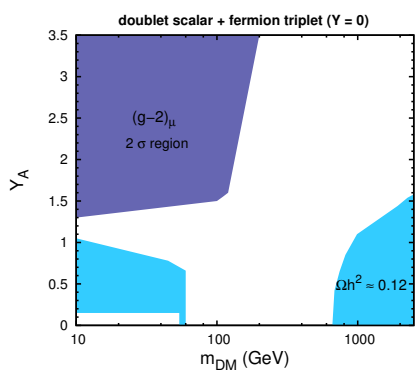

(c)

Figure 3: Planes of the Yukawa coupling to muons versus the dark matter mass in models with a scalar doublet. The region where the $(g-2) \mu$ anomaly can be accommodated at $2 \sigma$ is shown in blue, the region where the relic abundance is correct within $2 \sigma$ is shown in cyan, and the combined parameter space is highlighted in green. (a) Case 3a of Sec. 1.1, (b) Case 3c, (c) Case 3d.

in the theory and they can mix through the Higgs boson field vev (Case 1c). The former case does not show significant differences from Case 1a [5], whereas in the latter the mixing term introduces an additional explicit source of chiral-symmetry violation in the $(g-2)_{\mu}$ loop. As $\left|g_{p}\right| \ll 1$ in Eq. (1.2), one then obtains viable parameter space for large mass values, $m_{s}, m_{E} \sim \mathscr{O}(\mathrm{TeV})$, and small Yukawa couplings. For the same reason, however, Case $1 \mathrm{c}$ is subject to strong constraints from EWPOs, which can be used in the future to test these models.

\section{Models with a scalar SU(2) doublet}

The parameter space is more constrained by the relic density in cases with an SU(2) scalar doublet, as shown by the cyan region in the panels of Fig. 3. When considering the parameter space in agreement with $\delta(g-2)_{\mu}$, typical dark matter mass ranges from $m_{\mathrm{DM}} \approx 40 \mathrm{GeV}$ to the Higgs-resonance, $m_{\mathrm{DM}} \approx m_{h} / 2$. In the case with SU(2) triplet fermions (Case $3 \mathrm{c}$ of Sec. 1.1) the well-known presence of a doubly charged lepton in the spectrum enhances the calculation of $\delta(g-2)_{\mu}$, and since the Yukawa can be smaller the region with good relic density becomes confined to the Higgs resonance. In the case with SU(2) adjoint fermions (Case 3d) there is no

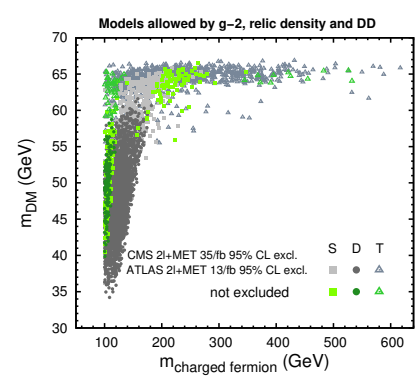

Figure 4: The impact of $\mathrm{LHC} 13 \mathrm{TeV}$ on the parameter space in agreement with dark matter and the $(g-2)_{\mu}$ anomaly in Models 4 (squares), 5 (circles), and 7 (triangles). The points allowed are shown in three different shades of green, while those excluded in three different shades of gray. 
viable parameter space where the BNL $\delta(g-2)_{\mu}$ and $\Omega h^{2} \approx 0.12$ can be obtained at the same time. Figure 4 shows that, in all models, most of the parameter space is almost entirely tested by LHC 2-lepton searches, except for the region of compressed spectra, where the new fermions and scalars are almost degenerate.

\section{Conclusions}

We have analyzed the impact of the $(g-2)_{\mu}$ measurement and the relic density of dark matter on the parameter space of simplified models of new physics invariant under $\mathrm{SU}(2) \times \mathrm{U}(1)$. The LHC imposes strong constraints on most models, with the exception of those featuring chiralityflip effects that can enhance the $(g-2)_{\mu}$ calculation. The latter models can be probed, however, by complementary means like future precision tests and direct detection of dark matter searches.

\section{References}

[1] G. W. Bennett et al. [Muon g-2 Collaboration], "Final Report of the Muon E821 Anomalous Magnetic Moment Measurement at BNL,” Phys. Rev. D 73, 072003 (2006) doi:10.1103/PhysRevD.73.072003 [hep-ex/0602035].

[2] M. Davier, "Update of the Hadronic Vacuum Polarisation Contribution to the muon g-2," Nucl. Part. Phys. Proc. 287-288, 70 (2017) doi:10.1016/j.nuclphysbps.2017.03.047 [arXiv:1612.02743 [hep-ph]].

[3] A. Chapelain [Muon g-2 Collaboration], “The Muon g-2 experiment at Fermilab,” EPJ Web Conf. 137, 08001 (2017) doi:10.1051/epjconf/201713708001 [arXiv:1701.02807 [physics.ins-det]].

[4] M. Otani [E34 Collaboration], "Status of the Muon g-2/EDM Experiment at J-PARC (E34)," JPS Conf. Proc. 8, 025008 (2015). doi:10.7566/JPSCP.8.025008

[5] K. Kowalska and E. M. Sessolo, "Expectations for the muon g-2 in simplified models with dark matter,” JHEP 1709, 112 (2017) doi:10.1007/JHEP09(2017)112 [arXiv:1707.00753 [hep-ph]].

[6] F. Jegerlehner and A. Nyffeler, “The Muon g-2,” Phys. Rept. 477, 1 (2009) doi:10.1016/j.physrep.2009.04.003 [arXiv:0902.3360 [hep-ph]].

[7] F. Bishara, J. Brod, P. Uttayarat and J. Zupan, "Nonstandard Yukawa Couplings and Higgs Portal Dark Matter,” JHEP 1601, 010 (2016) doi:10.1007/JHEP01(2016)010 [arXiv:1504.04022 [hep-ph]].

[8] E. Aprile et al. [XENON Collaboration], "First Dark Matter Search Results from the XENON1T Experiment," arXiv:1705.06655 [astro-ph.CO].

[9] The ATLAS collaboration [ATLAS Collaboration], "Search for supersymmetry with two and three leptons and missing transverse momentum in the final state at $\sqrt{s}=13 \mathrm{TeV}$ with the ATLAS detector," ATLAS-CONF-2016-096.

[10] CMS Collaboration [CMS Collaboration], "Search for new physics in events with two low momentum opposite-sign leptons and missing transverse energy at $\sqrt{s}=13 \mathrm{TeV}$," CMS-PAS-SUS-16-048.

[11] J. Erler, S. Heinemeyer, W. Hollik, G. Weiglein and P. M. Zerwas, "Physics impact of GigaZ," Phys. Lett. B 486, 125 (2000) doi:10.1016/S0370-2693(00)00749-8 [hep-ph/0005024].

[12] M. Bicer et al. [TLEP Design Study Working Group], "First Look at the Physics Case of TLEP," JHEP 1401, 164 (2014) doi:10.1007/JHEP01(2014)164 [arXiv:1308.6176 [hep-ex]]. 